\title{
On some distributions associated to boundary value problems
}

\author{
V. B. Vasilyev \\ Chair of Differential Equations, Belgorod National Research University, Belgorod, Russía
}

\section{ABSTRACT}

We consider an elliptic pseudo-differential equation in a multidimensional cone in Sobolev-Slobodetskii space and describe its general solution for some cases. This description is based on some distributions supported on a conical surface. For certain conical surfaces we describe such distributions. It permits to use such distributions to refine a general solution.

\section{ARTICLE HISTORY}

Received 21 June 2018

Accepted 12 January 2019

COMMUNICATED BY

A. Soldatov

\section{KEYWORDS}

Pseudo-differential equation; elliptic symbol; cone; wave factorization; general solution; distribution

\section{AMS SUBJECT} CLASSIFICATIONS

Primary: 35S15; Secondary:

$42 \mathrm{~B} 37 ; 46 \mathrm{~F} 10$

\section{Introduction}

Earlier the author has studied model elliptic pseudo-differential equations in canonical non-smooth domains in Euclidean space $\mathbb{R}^{m}$, and more completed results were obtained in the plane $m=2$. This approach is based on a special factorization of an elliptic symbol and leads to full description of solvabllity picture for a model elliptic pseudo-differential equation [1]. To transfer these results to multidimensional case one needs to know a general form of a distribution supported in a singular surface, this is the mam difficulty. There are a lot of examples for distributions supported on different types of surfaces in $m$-dimensional space [2], but there is no theorem on a general form of such a distribution. The author's latest studies related to the multidimensional situations $m \geq 3$ are very dependent on such a result. Some prelimmary results in this direction were obtained in papers [3-5], but here we will give more concrete calculations.

One can find other studies of pseudo-differential equations and related boundary value problems, for example, in $[6,7]$ and subsequent publications. 


\section{Preliminaries}

We consider the pseudo-differential operator $A$ :

$$
u(x) \longmapsto \int_{\mathbb{R}^{m}} \int_{\mathbb{R}^{m}} A(\xi) u(y) \mathrm{e}^{\mathrm{i}(y-x) \cdot \xi} \mathrm{d} \xi \mathrm{d} y, \quad x \in \mathbb{R}^{m}
$$

in the Sobolev-Slobodetskii spaces $H^{s}\left(\mathbb{R}^{m}\right)$ with norm

$$
\|u\|_{s}^{2}=\int_{\mathbb{R}^{m}}|\tilde{u}(\xi)|^{2}(1+|\xi|)^{2 s} \mathrm{~d} \xi, \quad \tilde{u}(\xi) \equiv(F u)(\xi)=\int_{\mathbb{R}^{m}} \mathrm{e}^{\mathrm{i} x \cdot \xi} u(x) \mathrm{d} x,
$$

and introduce the following class of symbols not depending on the spatial variable $x$ : $\exists c_{1}, c_{2}>0$ such that

$$
c_{1} \leq\left|A(\xi)(1+|\xi|)^{-\alpha}\right| \leq c_{2}, \quad \xi \in \mathbb{R}^{m}
$$

We call the number $\alpha \in \mathbb{R}$ order of the pseudo-differential operator $A$.

It is well known that such an operator is a linear bounded operator acting from the space $H^{s}\left(\mathbb{R}^{m}\right)$ into the space $H^{s-\alpha}\left(\mathbb{R}^{m}\right)$ and invertible [8]. The problem is the following: what can one say on the invertibility of the operator:

$$
u(x) \longmapsto \int_{D} \int_{\mathbb{R}^{m}} A(\xi) u(y) \mathrm{e}^{\mathrm{i}(y-x) \cdot \xi} \mathrm{d} \xi \mathrm{d} y, \quad x \in D,
$$

where $D \subset \mathbb{R}^{m}$ is a so-called 'canonical' domain. For the first canonical domam $D=$ $\mathbb{R}_{+}^{m} \equiv\left\{x \in \mathbb{R}^{m}: x=\left(x^{\prime}, x_{m}\right), x_{m}>0\right\}$ it was studied by M.I. Vishik-G.I. Eskin [8]. Here we consider another canonical domam, namely $D=C_{+}^{a}$,

$$
C_{+}^{a}=\left\{x \in \mathbb{R}^{m}: x_{m}>a\left|x^{\prime}\right|, x^{\prime}=\left(x_{1}, \ldots, x_{m-1}\right), a>0\right\} .
$$

Thus we study the solvability of the model pseudo-differential equation $[1,3,4]$

$$
\left(A u_{+}\right)(x)=f(x), \quad x \in C_{+}^{a},
$$

in the space $H^{s}\left(C_{+}^{a}\right)$, where $A$ is a pseudo-differential operator with the symbol $A(\xi)$ satisfymg the condition (1). The sign ' + ' for the unknown function $u_{+}$shows that this function is defined in $C_{+}^{a}$ only. We will recall some standard definitions.

By definition the space $H^{s}\left(C_{+}^{a}\right)$ consists of functions from the space $H^{s}\left(\mathbb{R}^{m}\right)$ with supports in $\overline{C_{+}^{a}}$. The norm in the space $H^{s}\left(C_{+}^{a}\right)$ is induced by the norm of the space $H^{s}\left(\mathbb{R}^{m}\right)$.

Let us denote by $S\left(\mathbb{R}^{m}\right)$ the $S$ chwartz class of imfinitely differentiable at mfinity rapidly decreasing functions, by $S^{\prime}\left(\mathbb{R}^{m}\right)$ the space of distributions over $S\left(\mathbb{R}^{m}\right)$, and by $S^{\prime}\left(C_{+}^{a}\right)$ the space of distributions from $S^{\prime}\left(\mathbb{R}^{m}\right)$ with supports in $\overline{C_{+}^{a}}$. The right-hand side $f$ in Equation (2) is taken from the space $H_{0}^{s-\alpha}\left(C_{+}^{a}\right)$ consisting of distributions from $S^{\prime}\left(C_{+}^{a}\right)$ which admit a continuation into the whole space $H^{s-\alpha}\left(\mathbb{R}^{m}\right)$. A norm in the space $H_{0}^{s-\alpha}\left(C_{+}^{a}\right)$ is defined by the formula

$$
\|f\|_{s-\alpha}^{+}=\inf \|l f\|_{s-\alpha},
$$

where infimum is taken over all continuations $l f$. 
Furthermore, we introduce the multidimensional singular integral operator:

$$
\left(G_{m} u\right)(x)=\lim _{\tau \rightarrow 0+} \int_{\mathbb{R}^{m}} \frac{u\left(y^{\prime}, y_{m}\right) \mathrm{d} y^{\prime} \mathrm{d} y_{m}}{\left(\left|x^{\prime}-y^{\prime}\right|^{2}-a^{2}\left(x_{m}-y_{m}+\mathrm{i} \tau\right)^{2}\right)^{m / 2}}
$$

(we omit certaim constants, see [1]). We recall that this integral is a multidimensional analogue of the Cauchy type integral; more exactly it is a variant of multidimensional Hilbert transform.

To introduce the next definition we add a new notation.

Let $C_{+}^{*}$ be the conjugate cone for the cone $C_{+}^{a}$ :

$$
\stackrel{*}{C_{+}^{a}}=\left\{x \in \mathbb{R}^{m}: x=\left(x^{\prime}, x_{m}\right), a x_{m}>\left|x^{\prime}\right|\right\}
$$

$C_{-}^{a} \equiv-C_{+}^{a}, T\left(C_{+}^{a}\right)$ be the radial tube domain over the cone $C_{+}^{a}$, i.e. a domain of the multidimensional complex space $\mathbb{C}^{m}$ of the type $\mathbb{R}^{m}+\mathrm{i} C_{+}^{a}$. The sign $\sim$ is used for the Fourier transform, so $\tilde{u}_{+}$is the Fourier transform of the function $u_{+}$, and $\tilde{H}$ is the Fourier image of the space $H$.

Definition 2.1: The wave factorization of an elliptic symbol $A(\xi)$ is called its representation in the form

$$
A(\xi)=A_{\neq}(\xi) A_{=}(\xi),
$$

where the factors $A_{\neq}(\xi), A_{=}(\xi)$ must satisfy the conditions:

(1) $A_{\neq}(\xi), A_{=}(\xi)$ are defined for all $\xi \in \mathbb{R}^{m}$ may be except $\left\{\xi \in \mathbb{R}^{m}:\left|\xi^{\prime}\right|^{2}=a^{2} \xi_{m}^{2}\right\}$;

(2) $A_{\neq}(\xi), A_{=}(\xi)$ admits an analytic contmuation into the radial tube domam $T\left(\stackrel{*}{C_{+}^{a}}\right), T\left(\stackrel{*}{C_{-}^{a}}\right)$, respectively with estimates

$$
\begin{aligned}
& \quad\left|A_{\neq}^{ \pm 1}(\xi+\mathrm{i} \tau)\right| \leq c_{1}(1+|\xi|+|\tau|)^{ \pm æ}, \\
& \quad\left|A_{=}^{ \pm 1}(\xi-\mathrm{i} \tau)\right| \leq c_{2}(1+|\xi|+|\tau|)^{ \pm(\alpha-æ)}, \quad \forall \tau \in C_{+}^{a} .
\end{aligned}
$$

The number $æ \in \mathbb{R}$ is called the index of the wave factorization.

Here we will consider Equation (2) for the case $æ-s=n+\delta, n \in \mathbf{N},|\delta|<1 / 2$, only. Let us note that for the case $æ-s=\delta$ the solution of Equation (2) is unique, and it can be reconstructed by the operator $G_{m}$ [1]. The general solution for our case can be constructed using the following scheme. We continue $f$ on the entire $\mathbb{R}^{m}$ and denote this continuation by lf. We put

$$
u_{-}(x)=l f(x)-\left(A u_{+}\right)(x)
$$

and rewrite Equation (2) as

$$
\left(A u_{+}\right)(x)+u_{-}(x)=l f(x) .
$$


Now applying the Fourier transform and the wave factorization we can write the initial equation in the form

$$
A_{\neq}(\xi) \tilde{u}_{+}(\xi)+A_{=}^{-1}(\xi) \tilde{u}_{-}(\xi)=A_{=}^{-1}(\xi) \tilde{l f}(\xi)
$$

Let us note that the function $A_{=}^{-1}(\xi) \tilde{l f}(\xi)$ belongs to the space $\tilde{H}^{s-\mathfrak{x}}\left(\mathbb{R}^{m}\right)$. So, if we take an arbitrary polynomial $Q(\xi)$ satisfying the condition

$$
|Q(\xi)| \sim(1+|\xi|)^{n},
$$

then the function $Q^{-1}(\xi) A_{=}^{-1}(\xi) \tilde{l f}(\xi)$ will belong to the space $\tilde{H}^{-\delta}\left(\mathbb{R}^{m}\right)$.

Furthermore, according to the theory of the multidimensional Riemann problem [1] we can represent the latter function as a sum of two summands, this is a so-called jump problem which can be solved by the operator $G_{m}$ :

$$
Q^{-1} A_{=}^{-1} \tilde{l f}=f_{+}+f_{-}
$$

where $f_{+} \in \tilde{H}^{-\delta}\left(C_{+}^{a}\right), f_{-} \in \tilde{H}^{-\delta}\left(\mathbb{R}^{m} \backslash C_{+}^{a}\right)$,

$$
f_{+}=G_{m}\left(A_{=}^{-1} \tilde{l f}\right), \quad f_{-}=\left(I-G_{m}\right)\left(A_{=}^{-1} \tilde{l f}\right) .
$$

Multiplying the equality (3) by $Q^{-1}(\xi)$ we rewrite it in the form

$$
Q^{-1} A_{\neq} \bar{u}_{+}+Q^{-1} A_{=}^{-1} \tilde{u}_{-}=f_{+}+f_{-},
$$

or

$$
Q^{-1} A_{\neq} \tilde{u}_{+}-f_{+}=f_{-}-Q^{-1} A_{=}^{-1} \tilde{u}_{-} .
$$

In other words

$$
A_{\neq} \tilde{u}_{+}-Q f_{+}=Q f_{-}-A_{=}^{-1} \tilde{u}_{-}
$$

Now we can use the following result $[1,5]$.

Lemma 2.2: If $\tilde{u}_{-} \in \tilde{H}^{s}\left(\mathbb{R}^{m} \backslash C_{+}^{a}\right), A_{=}^{-1}$ is a factor of the wave factorization then $A_{=}^{-1} \tilde{u}_{-} \in$ $\widetilde{H}^{s-\alpha+\infty}\left(\mathbb{R}^{m} \backslash C_{+}^{a}\right)$.

The left-hand side of the equality (4) belongs to the space $\bar{H}^{-n-\delta}\left(C_{+}^{a}\right)$, but the righthand side belongs to the space $\tilde{H}^{-n-\delta}\left(\mathbb{R}^{m} \backslash C_{+}^{a}\right)$. Therefore, we have

$$
F^{-1}\left(A_{\neq} \tilde{u}_{+}-Q f_{+}\right)=F^{-1}\left(Q f_{-}-A_{=}^{-1} \tilde{u}_{-}\right)
$$

where the left-hand side belongs to the space $H^{-n-\delta}\left(C_{+}^{a}\right)$, but right-hand side belongs to the space $H^{-n-\delta}\left(\mathbf{R}^{m} \backslash C_{+}^{a}\right)$, from which we conclude immediately that this is a distribution supported on the surface $\partial C_{+}^{a}$.

It is left to determime the form for such a distribution. 


\section{Distributions and change of variables}

\subsection{Test functions}

Let $C$ be a convex cone in the space $\mathbb{R}^{m}$, not including any entire straight line. This is important because we use the theory of analytic functions of several complex variables [9-11]. Moreover, we suppose that the surface of this cone is given by the equation $x_{m}=$ $\varphi\left(x^{\prime}\right), x^{\prime}=\left(x_{1}, \ldots, x_{m-1}\right)$, where $\varphi: \mathbb{R}^{m-1} \rightarrow \mathbb{R}$ is a smooth function in $\mathbb{R}^{m-1} \backslash\{0\}$, and $\varphi(0)=0$.

Let us introduce the change of variables:

$$
\begin{aligned}
t_{1} & =x_{1} \\
t_{2} & =x_{2} \\
\vdots & \\
t_{m-1} & =x_{m-1} \\
t_{m} & =x_{m}-\varphi\left(x^{\prime}\right)
\end{aligned}
$$

and denote this operator by $T_{\varphi}: \mathbb{R}^{m} \rightarrow \mathbb{R}^{m}$.

Obviously, this is a smooth transformation excluding the origm. We would like to define a special change of variables for distributions, and we need a special class of test functions. As such a space we take the Lizorkm space $\Phi\left(\mathbb{R}^{m}\right)$ [12] which is a subspace of the Schwartz space $S\left(\mathbb{R}^{m}\right)$. Such functions vanish at the origin with all their derivatives. If we denote by $\Phi^{\prime}\left(\mathbb{R}^{m}\right), S^{\prime}\left(\mathbb{R}^{m}\right)$ the correspondimg spaces of distributions then $\Phi^{\prime}\left(\mathbb{R}^{m}\right) \supset S^{\prime}\left(\mathbb{R}^{m}\right)$, and all operations with distributions from $\Phi^{\prime}\left(\mathbb{R}^{m}\right)$ will be valid for distributions from $S^{\prime}\left(\mathbb{R}^{m}\right)$.

Let $f$ be a local imtegrable function which generates a distribution defined by the formula

$$
(f, \psi)=\int_{\mathbb{R}^{m}} f(x) \psi(x) \mathrm{d} x
$$

We define a functional $T_{\varphi} f$ by the formula

$$
\left(T_{\varphi} f, \psi\right)=\left(f, T_{\varphi}^{-1} \psi\right)
$$

because

$$
\begin{aligned}
\left(T_{\varphi} f, \psi\right) & =\int_{\mathbb{R}^{m}}\left(T_{\varphi} f\right)(x) \psi(x) \mathrm{d} x \equiv \int_{\mathbb{R}^{m}} f\left(T_{\varphi} x\right) \psi(x) \mathrm{d} x \\
& =\int_{\mathbb{R}^{m}} f(x) \psi\left(T_{\varphi}^{-1} x\right) \mathrm{d} x=\left(f, T_{\varphi}^{-1} \psi\right) .
\end{aligned}
$$

One can easily verify that the Jacobian of the transformation $T_{\varphi}$ equals to 1 everywhere except 0 . Simce we choose such test functions the point 0 should be ignored.

We will use a result which follows from Schwartz theorem on one-dimensional distributions from $S^{\prime}(\mathbb{R})$ supported at the origm $0[2,10]$. 
Lemma 3.1: If a distribution $f \in S^{\prime}\left(\mathbb{R}^{m}\right)$ is supported in the hyper-plane $x_{m}=0$ then it has the form

$$
f(x)=\sum_{k=0}^{n} c_{k}\left(x^{\prime}\right) \otimes \delta^{(k)}\left(x_{m}\right), \quad x=\left(x^{\prime}, x_{m}\right),
$$

where $c_{k} \in S^{\prime}\left(\mathbb{R}^{m-1}\right), k=0,1, \ldots, n$, are arbitrary distributions.

\subsection{Change of variables}

Here we define a change of variables for distributions.

Definition 3.2: The operator $T_{\varphi}$ is defined by the formula

$$
\left(T_{\varphi} f, \psi\right)=\left(f, T_{\varphi}^{-1} \psi\right), \quad \forall \psi \in S\left(\mathbb{R}^{m}\right)
$$

The next two assertions are very simple.

Lemma 3.3: If a distribution $f \in S^{\prime}\left(\mathbb{R}^{m}\right)$ is supported on $\partial C$ then $T_{\varphi} f$ is supported on $\mathbb{R}^{m-1}$.

Theorem 3.4: An arbitrary distribution $f \in S^{\prime}\left(\mathbb{R}^{m}\right)$ supported on the conical surface $\partial C$ can be written in the form

$$
f(x)=T_{\varphi}^{-1}\left(\sum_{k=0}^{n} c_{k}\left(y^{\prime}\right) \otimes \delta^{(k)}\left(y_{m}\right)\right),
$$

where $c_{k} \in S^{\prime}\left(\mathbb{R}^{m-1}\right), k=0,1, \ldots, n$, are arbitrary distributions.

\section{The Fourier transform}

It is a natural way to ask what is the Fourier transform of a distribution supported on a conical surface. So, for example, the Fourier transform of the distribution (6) is a polynomial of order $n$ with respect to the variable $\xi_{m}$ with coefficients $\tilde{c}_{k}\left(\xi^{\prime}\right), k=0,1, \ldots, n$.

For functions $u(x)$ from $S\left(\mathbb{R}^{m}\right)$ the Fourier transform is defined by the formula

$$
(F u)(\xi) \equiv \tilde{u}(\xi)=\int_{\mathbb{R}^{m}} \mathrm{e}^{\mathrm{i} x \cdot \xi} u(x) \mathrm{d} x .
$$

The Fourier transform for distributions is defined as

$$
(F f, \psi)=(f, F \psi)
$$

therefore

$$
\left(F T_{\varphi} f, \psi\right)=\left(f, T_{\varphi}^{-1} F \psi\right)
$$

These properties imply that in order to define correlations between $T_{\varphi}$ and $F$ one can operate with test functions only. 
Let $f \in S^{\prime}\left(\mathbb{R}^{m}\right)$ be a distribution supported on $\partial C$. According to Theorem 3.4 it has the special form (6). We have the relations

$$
\begin{aligned}
(F f, \psi) & =\left(F T_{\varphi}^{-1}\left(\sum_{k=0}^{n} c_{k}\left(y^{\prime}\right) \otimes \delta^{(k)}\left(y_{m}\right)\right), \psi\right) \\
& =\left(F T_{\varphi}^{-1} F^{-1} F\left(\sum_{k=0}^{n} c_{k}\left(y^{\prime}\right) \otimes \delta^{(k)}\left(y_{m}\right)\right), \psi\right) \\
& =\left(V_{\varphi}\left(\sum_{k=0}^{n} \tilde{c}_{k}\left(\xi^{\prime}\right) \xi_{m}^{k}\right), \psi\right),
\end{aligned}
$$

where we use the new notation $F T_{\varphi}^{-1} F^{-1} \equiv V_{\varphi}$; this operator $V_{\varphi}$ is defined on $S\left(\mathbb{R}^{m}\right)$; let us note that this operator is imvertible and $V_{\varphi}^{-1}=F T_{\varphi} F^{-1}, T_{\varphi}^{-1}=T_{-\varphi}$. According to the latter observations we can conclude that

$$
F f=V_{\varphi}\left(\sum_{k=0}^{n} \tilde{c}_{k}\left(\xi^{\prime}\right) \xi_{m}^{k}\right)
$$

\subsection{The operator $V_{\varphi}$}

We start from the equality

$$
F T_{\varphi}^{-1}=V_{\varphi} F
$$

and conclude that this operator acts in Fourier images. It is more convenient to start from the left-hand side. We fix $u \in S\left(\mathbb{R}^{m}\right)$ and calculate:

$$
\begin{aligned}
\left(F T_{\varphi}^{-1} u\right)(\xi) & =\int_{\mathbb{R}^{m}} \mathrm{e}^{\mathrm{i} y \cdot \xi}\left(T_{\varphi}^{-1} u\right)(y) \mathrm{d} y=\int_{\mathbb{R}^{m}} \mathrm{e}^{\mathrm{i} y \cdot \xi} u\left(y^{\prime}, y_{m}+\varphi\left(y^{\prime}\right)\right) \mathrm{d} y \\
& =\int_{\mathbb{R}^{m}} \mathrm{e}^{\mathrm{i} x \cdot \xi} \mathrm{e}^{-\mathrm{i} \xi_{m} \varphi\left(x^{\prime}\right)} u\left(x^{\prime}, x_{m}\right) \mathrm{d} x^{\prime} \mathrm{d} x_{m}=\int_{\mathbb{R}^{m-1}} \mathrm{e}^{\mathrm{i} x^{\prime} \cdot \xi^{\prime}} \mathrm{e}^{-\mathrm{i} \xi_{m} \varphi\left(x^{\prime}\right)} \hat{u}\left(x^{\prime}, \xi_{m}\right) \mathrm{d} x^{\prime},
\end{aligned}
$$

where $\hat{u}\left(x^{\prime}, \xi_{m}\right)$ denotes the Fourier transform of the function $u\left(x^{\prime}, x_{m}\right)$ with respect to the variable $x_{m}$. Taking into account properties of the Fourier transform one can make the following conclusion. Let us denote

$$
F_{x^{\prime} \rightarrow \xi^{\prime}}\left(\mathrm{e}^{-1 \xi_{m} \varphi\left(x^{\prime}\right)}\right) \equiv K_{\varphi}\left(\xi^{\prime}, \xi_{m}\right)
$$

and after this we obtam an integral representation for the operator $V_{\varphi}$ :

$$
\left(F T_{\varphi}^{-1} u\right)(\xi)=\int_{\mathbb{R}^{m}} K_{\varphi}\left(\xi^{\prime}-\eta^{\prime}, \xi_{m}\right) \tilde{u}\left(\eta^{\prime}, \xi_{m}\right) \mathrm{d} \eta^{\prime}
$$




\subsection{A first example: $m=2$}

The case $m=2$ is very good, there is only one mentioned cone. We write it as

$$
C_{+}^{a}=\left\{x \in \mathbb{R}^{2}: x=\left(x_{1}, x_{2}\right), x_{2}>a\left|x_{1}\right|, a>0\right\}
$$

and further evaluate:

$$
\begin{aligned}
\left(F T_{\varphi} u\right)(\xi)= & \int_{-\infty}^{+\infty} \mathrm{e}^{\mathrm{i} a\left|y_{1}\right| \xi_{2}} \mathrm{e}^{\mathrm{i} y_{1} \xi_{1}} \hat{u}\left(y_{1}, \xi_{2}\right) \mathrm{d} y_{1}, \\
= & \int_{-\infty}^{+\infty} \chi_{+}\left(y_{1}\right) \mathrm{e}^{\mathrm{i} a y_{1} \xi_{2}} \mathrm{e}^{\mathrm{i} y_{1} \xi_{1}} \hat{u}\left(y_{1}, \xi_{2}\right) \mathrm{d} y_{1} \\
& +\int_{-\infty}^{+\infty} \chi-\left(y_{1}\right) \mathrm{e}^{-\mathrm{i} a y_{1} \xi_{2}} \mathrm{e}^{\mathrm{i} y_{1} \xi_{1}} \hat{u}\left(y_{1}, \xi_{2}\right) \mathrm{d} y_{1} \\
= & \int_{-\infty}^{+\infty} \chi_{+}\left(y_{1}\right) \mathrm{e}^{\mathrm{i} y_{1}\left(a \xi_{2}+\xi_{1}\right)} \hat{u}\left(y_{1}, \xi_{2}\right) \mathrm{d} y_{1} \\
& +\int_{-\infty}^{+\infty} \chi_{-}\left(y_{1}\right) \mathrm{e}^{-\mathrm{i} y_{1}\left(a \xi_{2}-\xi_{1}\right)} \hat{u}\left(y_{1}, \xi_{2}\right) \mathrm{d} y_{1},
\end{aligned}
$$

where $\hat{u}\left(y_{1}, \xi_{2}\right)$ denotes the one-dimensional Fourier transform with respect to the second variable, $\chi_{ \pm}$are indicators of $\mathbb{R}_{ \pm}$.

The latter two summands are Fourier transforms of functions

$$
\chi+\left(y_{1}\right) \hat{u}\left(y_{1}, \xi_{2}\right), \quad \chi-\left(y_{1}\right) \hat{u}\left(y_{1}, \xi_{2}\right)
$$

with respect to the variable $y_{1}$ so that we can use the following properties [8]; these properties are called Sokhotskii formulas $[13,14]$ :

$$
\begin{aligned}
& \int_{-\infty}^{+\infty} \chi_{+}(x) \mathrm{e}^{\mathrm{i} x \xi} u(x) \mathrm{d} x=\frac{1}{2} \tilde{u}(\xi)+v \cdot p \cdot \frac{\mathrm{i}}{2 \pi} \int_{-\infty}^{+\infty} \frac{\tilde{u}(\eta) \mathrm{d} \eta}{\xi-\eta} \\
& \int_{-\infty}^{+\infty} \chi-(x) \mathrm{e}^{\mathrm{i} x \xi} u(x) \mathrm{d} x=\frac{1}{2} \tilde{u}(\xi)-v \cdot p \cdot \frac{\mathrm{i}}{2 \pi} \int_{-\infty}^{+\infty} \frac{\tilde{u}(\eta) \mathrm{d} \eta}{\xi-\eta}
\end{aligned}
$$

Taking these properties imto account we conclude

$$
\begin{aligned}
\left(F T_{\varphi}^{-1} u\right)(\xi)= & \frac{\tilde{u}\left(\xi_{1}+a \xi_{2}, \xi_{2}\right)+\tilde{u}\left(\xi_{1}-a \xi_{2}, \xi_{2}\right)}{2} \\
& +v \cdot p \cdot \frac{\mathrm{i}}{2 \pi} \int_{-\infty}^{+\infty} \frac{\tilde{u}\left(\eta, \xi_{2}\right) \mathrm{d} \eta}{\xi_{1}+a \xi_{2}-\eta}-v \cdot p \cdot \frac{\mathrm{i}}{2 \pi} \int_{-\infty}^{+\infty} \frac{\tilde{u}\left(\eta, \xi_{2}\right) \mathrm{d} \eta}{\xi_{1}-a \xi_{2}-\eta} \equiv\left(V_{\varphi} \tilde{u}\right)(\xi) .
\end{aligned}
$$

\subsection{An example: $m \geq 3$}

As it was shown the kernel $K_{\varphi}$ is computable for concrete function $\varphi\left(x^{\prime}\right)$. Let $\varphi\left(x^{\prime}\right)=a\left|x^{\prime}\right|$, $a>0$. If we will look at the formulas from [11] (see also [15] in which a real analogue of 
these formulas is given as the Poisson kernel) we will find

$$
K_{\varphi}\left(\xi^{\prime}, \xi_{m}\right)=\frac{a 2^{m-1} \pi^{(m-2) / 2} \Gamma(m / 2)}{\left(\xi_{1}^{2}+\xi_{2}^{2}+\cdots \xi_{m-1}^{2}-a^{2} \xi_{m}^{2}\right)^{m / 2}} .
$$

Therefore for such a multidimensional cone the operator $V_{\varphi}$ looks like

$$
\left(V_{\varphi} \tilde{u}\right)(\xi)=\int_{\mathbb{R}^{m-1}} \frac{a 2^{m-1} \pi^{(m-2) / 2} \Gamma(m / 2) \tilde{u}\left(\eta^{\prime}, \xi_{m}\right) \mathrm{d} \eta^{\prime}}{\left(\left(\xi_{1}-\eta_{1}\right)^{2}+\left(\xi_{2}-\eta_{2}\right)^{2}+\cdots\left(\xi_{m-1}-\eta_{m-1}\right)^{2}-a^{2} \xi_{m}^{2}\right)^{m / 2}} .
$$

In our opinion we could call it a conical potential.

Remark 4.1: Of course the formula (7) should be treated in the distributional sense. Below we give such a definition for the operator $V_{\varphi}$ in the space $S^{\prime}\left(\mathbb{R}^{m}\right)$.

Definition 4.1: For a distribution $f \in S^{\prime}\left(\mathbb{R}^{m}\right)$ the transform $V_{\varphi}$ is given by the formula

$$
\left(V_{\varphi} \tilde{f}, \psi\right) \equiv\left(\tilde{f}, V_{-\varphi} \psi\right), \quad \forall \psi \in S\left(\mathbb{R}^{m}\right) .
$$

\section{The operator $V_{\varphi}$ and a general solution}

Let $C$ be a convex cone not imcluding an entire straight line. Let us introduce the Bochner kernel [9-11]

$$
B_{m}(z)=\int_{C} \mathrm{e}^{\mathrm{i} x \cdot z} \mathrm{~d} x, \quad z=\xi+\mathrm{i} \tau,
$$

and the related integral operator

$$
\left(B_{m} u\right)(x)=\lim _{\tau \rightarrow 0+} \int_{\mathbb{R}^{m}} B_{m}(x-y+\mathrm{i} \tau) u(y) \mathrm{d} y, \quad x \in \mathbb{R}^{m} .
$$

The reader can easily adapt Definition 2.1 for an arbitrary cone $C=\left\{x \in \mathbb{R}^{m}: x_{m}>\varphi\left(x^{\prime}\right)\right\}$ and he can obtam the following result for Equation (2) in a cone $C$.

Theorem 5.1: If the symbol $A(\xi)$ admits the wave factorization with the index $æ, \rightsquigarrow-s=$ $n+\delta, n \in \mathbb{N},|\delta|<1 / 2$, then the general solution of Equation (2) in Fourier images is given by the formula

$$
\begin{aligned}
\tilde{u}_{+}(\xi)= & A_{\neq}^{-1}(\xi) Q(\xi) B_{m} Q^{-1}(\xi) A_{=}^{-1}(\xi) \tilde{l f}(\xi) \\
& +A_{\neq}^{-1}(\xi) V_{\varphi}^{-1} F\left(\sum_{k=1}^{n} c_{k}\left(x^{\prime}\right) \delta^{(k-1)}\left(x_{m}\right)\right),
\end{aligned}
$$

where $c_{k}\left(x^{\prime}\right) \in H^{s_{k}}\left(\mathbb{R}^{m-1}\right)$ are arbitrary functions, $s_{k}=s-æ+k-1 / 2, k=1,2, \ldots, n, l f$ is an arbitrary continuation off onto $H^{s-\alpha}\left(\mathbb{R}^{m}\right)$.

The corresponding result on a general solution of Equation (2) in the cone $C_{+}^{a}$ was proved in [5]. 
Remark 5.1: It is very simple to verify that the result does not depend on the contimuation lf $[1,8]$.

Some special cases are very imterestimg, for example if $C=C_{+}^{a}$.

Corollary 5.2: Iff $\equiv 0, n=1$, then we have the following form for the general solution in the space $H^{s}\left(C_{+}^{a}\right)$ :

$$
\tilde{u}_{+}(\xi)=A_{\neq}^{-1}(\xi) \int_{\mathbb{R}^{m-1}} \frac{a 2^{m-1} \pi^{(m-2) / 2} \Gamma(m / 2) \tilde{c}\left(\eta^{\prime}\right) \mathrm{d} \eta^{\prime}}{\left(\left(\xi_{1}-\eta_{1}\right)^{2}+\left(\xi_{2}-\eta_{2}\right)^{2}+\cdots\left(\xi_{m-1}-\eta_{m-1}\right)^{2}-a^{2} \xi_{m}^{2}\right)^{m / 2}},
$$

where $c\left(x^{\prime}\right) \in H^{s-æ+1 / 2}\left(\mathbb{R}^{m-1}\right)$ is an arbitrary function.

Using these results one needs to add some additional conditions to determme uniquely the unknown functions $c_{k}$. We will consider this problem in forthcoming papers.

\section{Acknowledgments}

The author is very grateful to referees for their timely and helpful comments.

\section{Disclosure statement}

No potential conflict of interest was reported by the author.

\section{ORCID}

V. B. Vasilyev (1D http://orcid.org/0000-0001-9351-8084

\section{References}

[1] Vasilev VB. Wave factorization of elliptic symbols: theory and applications. Introduction to the theory of boundary value problems in non-smooth domains. Dordrecht, Boston, London: Kluwer Academic Publishers; 2000.

[2] Gel'fand IM, Shilov GE. Generalized functions. Vol. 1. Properties and operations. New York, London: Academic Press; 1964.

[3] Vasilyev VB. On the Dirichlet and Neumann problems in multi-dimensional cone. Math Bohem. 2014;139(2):333-340.

[4] Vasilyev VB. On certain elliptic problems for pseudo differential equations in a polyhedral cone. Adv Dyn Syst Appl. 2014;9(2):227-237.

[5] Vasilyev VB. Potentials for elliptic boundary value problems in cones. Sib Elektron Mat Izv. 2016;13:1129-1149 (Russian).

[6] Nazarov SA, Plamenevsky BA. Elliptic problems in domains with piecewise smooth boundaries. Berlin, New York: Walter de Gruyter; 1994.

[7] Schulze B-W, Sternin B, Shatalov V. Differential equations on singular manifolds: semiclassical theory and operator algebras. Berlin: Wiley-VCH; 1998.

[8] Eskin G. Boundary value problems for elliptic pseudodifferential equations. Providence: American Mathematical Society; 1981.

[9] Bochner S, Martin WT. Several complex variables. Princeton: Princeton University Press; 1948.

[10] Vladimirov VS. Methods of the theory of functions of many complex variables. Mineola: Dover Publications; 2007. 